\title{
Harmonization of Semantic Data Models of Electric Data Standards
}

\author{
Juan Carlos Nieves*, Mariano Ortega de Mues $^{\dagger}$, Angelina Espinoza ${ }^{\ddagger}$, Daniel Rodríguez-Álvarez ${ }^{\S}$ \\ * Software Department (LSI), Technical University of Catalonia \\ c/Jordi Girona 1-3, E08034, Barcelona Spain \\ ASCAMM - Technology Center, Parc Tecnològic del Vallès, Cerdanyola del Vallès, Spain \\ Email: jcnieves@1si.upc.edu \\ †Indra Sistemas, Energy Market Department \\ Avda. de Bruselas 35, E-28108, Madrid, Spain \\ Email: mortega@indra.es \\ † Technical University of Madrid (UPM) \\ Ctra. de Valencia Km. 7, E-28031, Madrid, Spain \\ Email: a.espinoza@syst.eui.upm.es \\ $\S$ INDRA Software Labs \\ C. Acanto, 11, E-28045 \\ Madrid, Spain \\ Email:dralvarez@indra.es
}

\begin{abstract}
According to the Electric Power Research Institute (EPRI) a common semantics model is necessary for achieving interoperability in the Smart Grid vision. In this paper, we present an outline of two influential International Electrotechnical Commission Standards (CIM and IEC 61850) for building a common semantic model in a Smart Grid vision. In addition, we revise two representative approaches suggested by EPRI for harmonizing these standards in a common semantic model. The pros and cons between these two approaches are identified and analyzed.
\end{abstract}

\section{INTRODUCTION}

Smart Grid initiatives present an unprecedented opportunity for the energy industry to serve its customers with a new generation of efficient and reliable products and services. The Smart Grid is characterized by a two-way flow of electricity and information to create an automated, widely distributed energy delivery network. It incorporates into the grid the benefits of distributed computing and communications to deliver real-time information and enable the near-instantaneous balance of supply and demand at the device level [1]. In this scenario, all grids' systems adopt different data standards depending on the underlying electrical application domain. Thus, interoperability is one of the major challenges in Smart Grids, as it is claimed by the National Institute of Standards and Technology (NIST), in [1], and the International Electrotechnical Commission (IEC) in [2].

Existing electric standards address different data interoperability levels, but they cover different domains with large overlaps among them. This situation presents a huge challenge for the harmonization and evolution of electric standards. The most common issues towards harmonization and evolution are:

- Differing levels of maturity,

- Overlapping focus areas and
- Different levels of physical implementation (application vs. device).

For addressing these problems, the NIST Roadmap [1] defines the need for a common semantic model based on the harmonization of different electric standards. This harmonization relies on the integration of several data standards. Fig. 1, taken from [3], shows the relationships among different electric industrial standards which can be considered relevant in the data domains of Smart Grids. From this figure, one can observe that the standards: IEC 61970, IEC 61968 and IEC 61850 have a big scope in the domains of smart grids. Hence, a harmonized semantic model based on these IEC standards has been the objective of an intensive research in the Electric Power Research Institute (EPRI) [4], [5]. However, it seems that there is not an ideal approach for building this harmonized semantic data model. Hence, some guidelines for selecting a particular approach for building a harmonized semantic data models based on IEC 61970, IEC 61968 and IEC 61850 are required.

The research presented in this paper is focused on some issues of the harmonization of the semantic date models of IEC 61970, IEC 61968 and IEC 61850. We start presenting an outline of IEC 61970, IEC 61968 and IEC 61850. In addition, we revise the two representative approaches suggested by EPRI for harmonizing these standards in a common semantic model [4], [5]. After this, the pros and cons between these two approaches are identified and analyzed. We argue that these results will help to support the decision of seleccting an appropriate approach for building a semantic data model of a particular Smart Grid.

The paper is structured as follows: In Section II some basic concepts in the context of semantic data models are introduced. Section III describes the model CIM. Section IV 


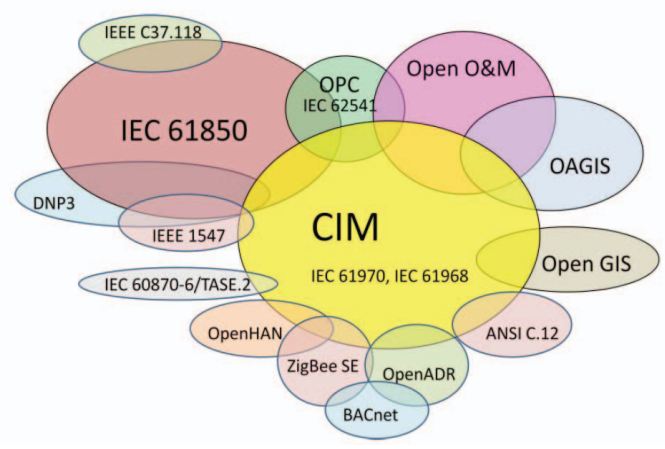

Fig. 1. Logical Relationships between Smart Grid Standards.

introduces the IEC 61850 SCL. In Section V, two approaches for harmonizing CIM and IEC 61850 SCL are described. Here, the properties of these approaches are also discussed. In the last section, the conclusions and future works are presented.

\section{BACKground: A Semantic Model}

The objective of this section is to give an intuitive idea of basic concepts which are relevant for this paper, e.g. a knowledge base, a semantic model, an ontology, etc.

We start describing the basic idea of a knowledge model. Basically, a knowledge model is a way to abstract disparate data and information. Knowledge modeling is about describing what data means and where it fits. It allows us to understand and abstract knowledge. Consequently it helps us to understand how different pieces of information relate to each other [6]. Usually a knowledge base is captured by declarative specification languages such as, First Order Logic, Description Logics, etc. [7], [8].

A semantic model is one kind of a knowledge model. The semantic model consists of a network of concepts and the relationships between those concepts. Concepts are a particular idea or topic with which the user is concerned. For instance the concept PowerSystemResoruce which according to CIM standard is defined as follows:
A power system resource can be an item of equip- ment such as a Switch, an EquipmentContainer containing many individual items of equipment such as a Substation, or an organisational entity such as Company or SubControlArea. This provides for the nesting of collections of PowerSystemResources within other PowerSystemResources. For example, a Switch could be a member of a Substation and a Substation could be a member of a division of a Company.

Notice that the concept of PowerSystemResources implicitly defines a class of resources which has relations to other concepts in the domain of electric systems, see Fig. 2.
In general terms, the concepts and relationships together are often known as an ontology; the semantic model that describes knowledge. The semantic models enable users to ask questions about the information in a natural way helping to identify patterns and trends in this information and also discovering relationships between disparate pieces of information.

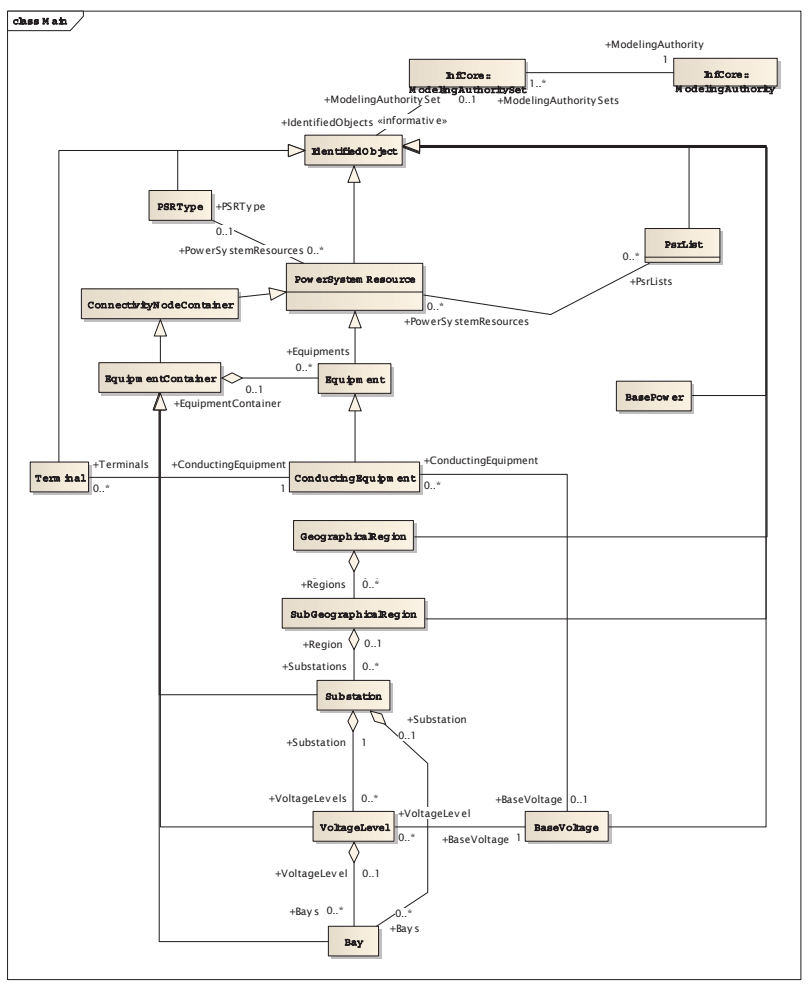

Fig. 2. UML diagram of the classes of package Core of the standard CIM version 14.

\section{CIM: COMMON INFORMATION MODEL}

The Common Information Model (CIM) is a standard for representing power system components developed by EPRI. CIM is formed by a set of standards suggested by EPRI; however, the standards IEC 61970-301 [9] and IEC-61968-11 [10] are known as the core of CIM.

- IEC 61970-301: The IEC standard 61970-301 is a semantic model that describes the components of a power system at an electrical level and the relationships between each component.

- IEC-61968-11: The IEC 61968-11 extends the semantics model of IEC 61970-301 to cover the other aspects of power system software data exchange such as asset tracking, work scheduling and customer billing.

The semantics data model of CIM is currently maintained as an UML model. This semantic model defines a common vocabulary and a basic ontology. This semantic model can be used to derive particular artifacts (e.g. , RDF schemas, OWL ontologies, SQL schemas, etc.) as needed. In the industrial 
be defined.

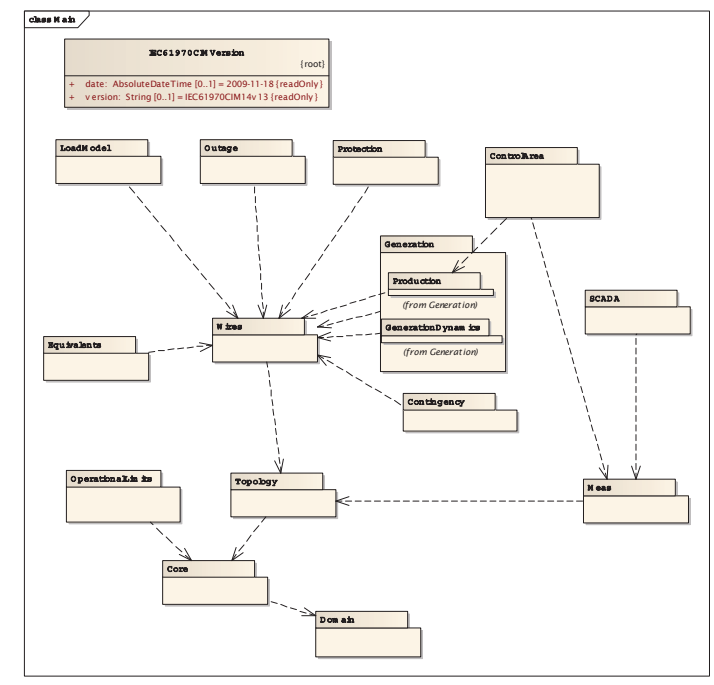

Fig. 3. Main packages of IEC 61970.

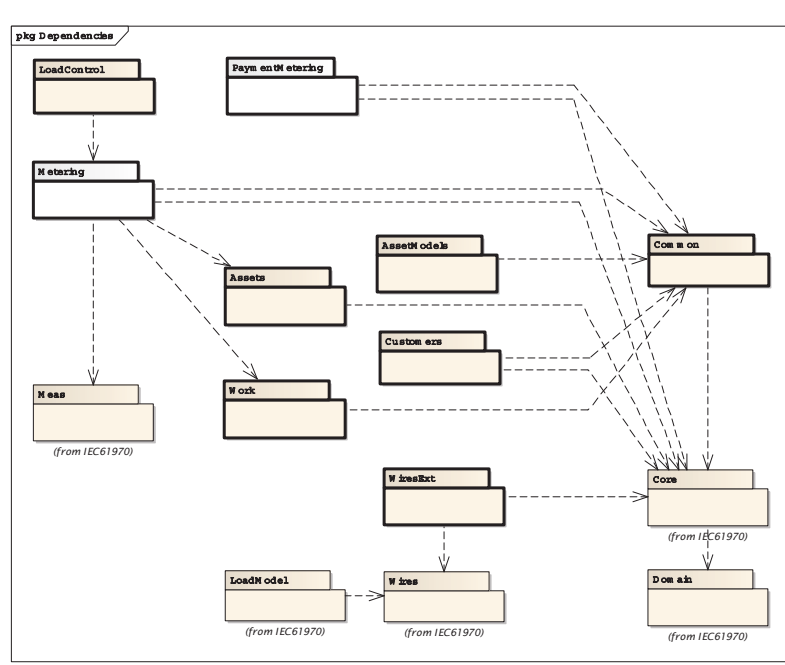

Fig. 4. Main packages of IEC 61968.

sector, one can find different systems for managing the semantic model of CIM e.g. , $\mathrm{CIMTool}^{1}, \mathrm{CIMvian}^{2}, \mathrm{CIMSpy}^{3}$, $\mathrm{OpenCIM}^{4}$. Some of these systems use OWL to specify subsets of the full semantic model, called profiles. Profiles are commonly defined in OWL, instead of UML, because it allows axioms that place further restrictions on the model to

\footnotetext{
${ }^{1}$ http://www.cimtool.org/

${ }^{2}$ http://www.uisol.com/uisol/CIMvian/CIMvian.htm

${ }^{3} \mathrm{http}$ ///www.powerinfo.us/opensource/download.html

${ }^{4}$ http://sites.google.com/a/interpss.com/opencim/
}

\section{IEC 61850}

IEC 61850 is another international standard developed by EPRI and supported by IEC. In particular IEC 61850 is a standard for the design of electric substation automation. One of the most interesting things of IEC 61850 is that the defined abstract data model can be mapped to different kinds of protocols. Some of those mappings are into MMS (Manufacturing Message Specification [11]), GOOSE (Generic Object Oriented Substation Events), SMV, and to Web Services [12]. It is important to point out that IEC 61850 is not only a communication protocol that describes the communication between Intelligent Electronic Devices (IED) and a SCADA ${ }^{5}$ system, it does also address many tasks inside a substation (usually this tasks are managed by the so called Substation Configuration Language SCL). It is worth mentioning that other standards and IEC working groups are now using the concept of the IEC 61850 for their own domain; it includes the DER, the WindPower and the Hydro working groups; the standard is therefore gaining influence in many domains of the power utility automation.

As we already mentioned, IEC 61850 allows addressing several tasks inside a substation by the SCL language. The SCL language is a specification language based on the eXtensible Markup Language (XML) to describe the configuration of IEC 61850-based systems. SCL specifies a hierarchy of configuration files that enable multiple levels of the system to be described in unambiguous and standardized XML files. The various SCL files include system specification description (SSD), IED capability description (ICD), substation configuration description (SCD), and configured IED description (CID) files. All these files are constructed in the same methods and format but have different scopes depending on the need [12], [13]. The Fig. 5 taken from [13] shows an UML diagram which gives an overview of how the SCL schema is structured.

We want to point out that the schema of Fig. 5 does not define a semantic data model of IEC 61850. In fact unlike to CIM, IEC 61850 has not a defined semantic data model. Hence, the definition of a semantic model of IEC 61850 requires analyzing the SCL schema for defining a semantic model of all the objects and properties of IEC 61850. As we can see in Fig. 5, a usual SCD SCL file is formed by five sections: Headers, Substation, Communication, IED, and DataTypeTemplates.

In Fig. 6 taken from [13], a simplified UML model of IEC 61850 is presented. Observe that the UML model presented in 6 is not a complete in the modelling sense. In other words, it does not show any superclasses from which the used classes may be derived, or any attributes. Essentially, UML model restricts itself to those concrete object types that are used with a SCL file.

\footnotetext{
${ }^{5}$ SCADA: Supervisory Control And Data Acquisition.
} 


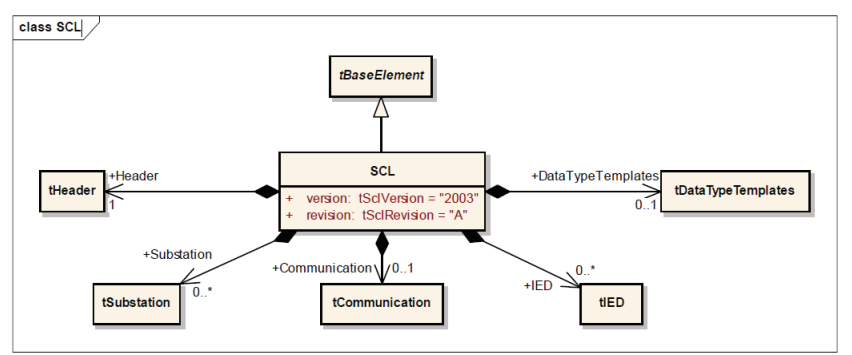

theoretically include any CIM data. These features suggest two main kinds of harmonizations [4]:

1) A harmonization for the configuration time which suggests issues such as how the information from IEC 61850 SCL information model maps to the CIM and how to document and manage this mapping.

2) A harmonization for the run time which suggest issues such as how the Field Device Communication Interface can be configured so that the view of the substation information model, seen by the Operations and Maintenance environment would always match the view found in substation devices.

In order to manage these harmonizations, different approaches has been proposed [4], [5]. Some of these approaches suggests to extended the actual semantic model of CIM for building the semantic model of IEC 61850 [5] (the harmonization in this case is managed by explicit extension of classes)

Fig. 5. UML diagram overview of SCL schema.

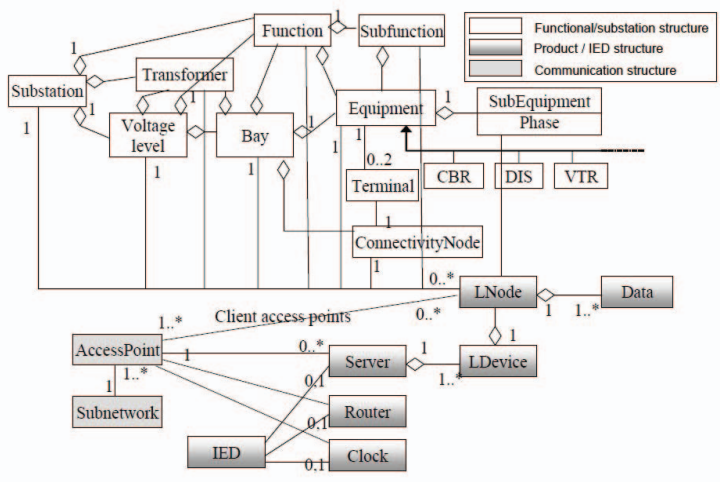

Fig. 6. UML - SCL Object Model

\section{Semantic Data Model Harmonization}

According to [4], there are three information models that are somewhat independent but need to be linked:

- The IEC 61968/61970 Common Information Model (CIM)

- The IEC 61850 Configuration Model (the SCL files)

- The IEC 61850 Run Time Model (the IEDs providing the logical nodes and data)

From this perspective, it is clear that we have to define relationships inside the CIM semantic model (Fig. 3 and 4) and the concepts of the SCL schema (5). For this end, we want to point out that IEC 61850 differs from CIM in that its configuration model, the SCL language, contains different information than its run-time model. On the other hand, in CIM, the same information may be presented in either timeframe. This means that, the CIM XML data model may and others suggest to build semantics models by using UML models and OWL axioms [4] (the harmonization in this case is managed by a OWL ontology approach). All of these proposals agree in the necessity of a semantic model for IEC 61850. For answering this necessity, the working group WG10 of EPRI is developing the official UML semantic data model of IEC $61850^{6}$. Hence, we can assume that soon we will have a basic ontology of the concepts of IEC 61850. Therefore one will be able to build a basic OWL ontology of IEC 61850 .

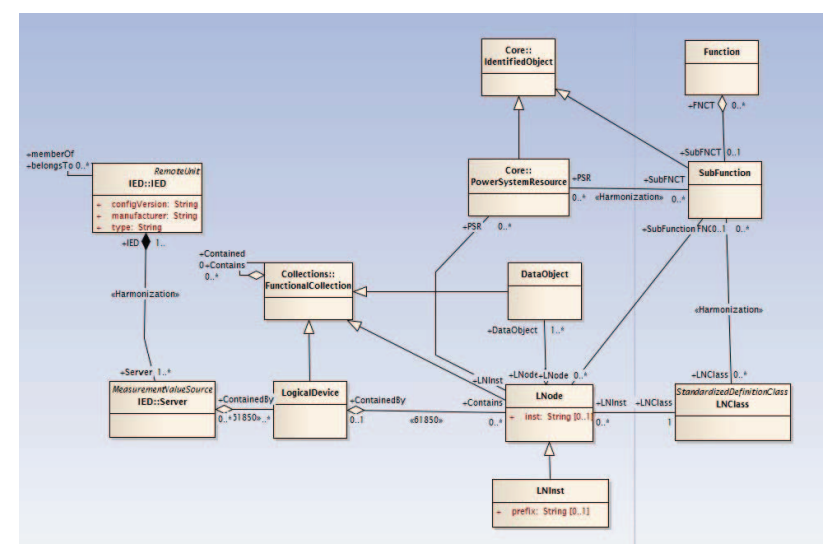

Fig. 7. An example of the harmonization between CIM and IEC 61850 by using a unified semantics model.

Possibly the best approach for harmonizing IEC 61850 and CIM depends on the context of each Smart Grid.

- On the one hand, to build a unified UML model which covers the domains of IEC 61850 and CIM could be a good solution for the case that there is already implementations of CIM-based systems. In this case, we are

${ }^{6}$ Talk: CIM and 61850 Harmonization, presented by Herbert Falk (SISCO), September 16, 2010 
assuming that the version of CIM is freezed. This means that the UML definitions of the concepts of CIM will not change with the time, Fig. 7 (this figure was taken from [5]).

- On the other hand, to made independent semantics models could be a good solution for the case that all the platforms of the Smart Grid will be designed from scratch. In this case, we are assuming that the harmonization is done by an OWL ontology approach (as it is done in [4]). Hence the integration of the semantic data models is managed by the explicit definition of equality OWL axioms between classes of IEC 61850 and CIM, Fig. 8 (this figure was taken from [4]).

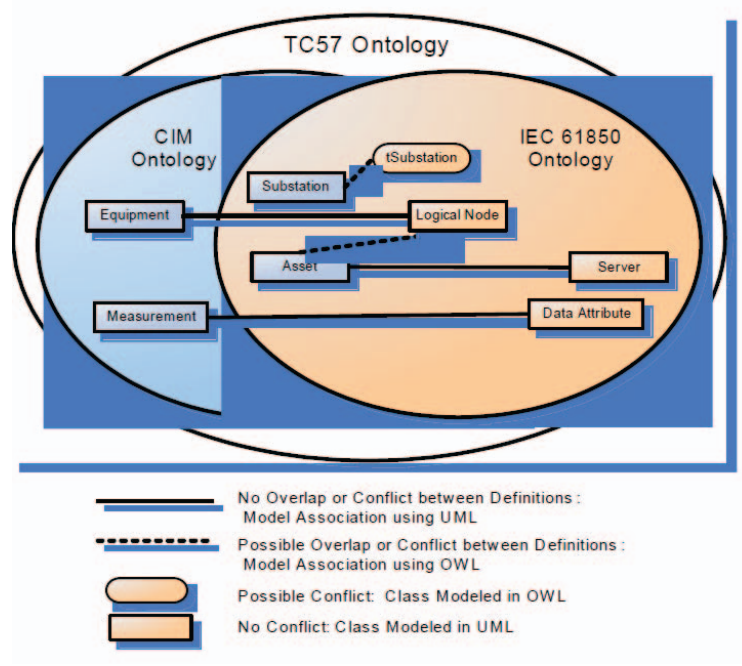

Fig. 8. Modeling and Mapping Ontology's.

One fundamental issue in the harmonization of IEC 61850 and CIM is the flexibility of the proposals for updating the semantic data models of each standard. This issue emerges by the constant delivering of versions of the semantic data model of $\mathrm{CIM}^{7}$. These changes are quite natural since the Energy Management Systems (EMS) constantly require the implementation of new functionalities. In fact, EPRI continues to sponsor research in areas where CIM needs additional definition or visibility such as the use of CIM for planning and dynamic modeling initiatives [14], [15].

In the harmonization approach of IEC 61850 and CIM by using a unified UML model (Fig. 7, [5]), the CIM version is freezed. This restriction makes rigid the semantic data model for updating the semantic data model of each standard. In this case, the process of updating the unified UML model requires redefining each class of the intersection of IEC 61850 and CIM. On the other hand, in the approach of independent

\footnotetext{
${ }^{7}$ Until today there are at least 14 versions of CIM, see: http://cimug.ucaiug.org/
}

semantics models, the process of updating the harmonized semantic model is restricted to update the equality OWL axioms which link the semantics models (OWL ontologies). This situation suggests that the process of updating the harmonized semantic model is flexible.

Another fundamental issue to consider in the harmonization is the support of the approach for handing subsets of the harmonized semantic model. These subsets usually are called profiles [15]. On the one hand, a profile makes possible to use a semantic model in a computable fashion. On the other hand, a profile makes restrictions which are mandatory for particular application schemas, e.g. an electric model, an XML message, a SQL schema, etc. By assuming that there is an unique UML model of the harmonization of IEC 61850 and CIM, one requires a unique profile of this semantic model for generating its application schemas. For instance, if there is a profile $\mathcal{P}$ which satisfies the restrictions of a SCL schema (Fig. 5), from $\mathcal{P}$ one can generate its corresponding SCL files. Observe that we are assuming that $\mathcal{P}$ is containing concepts from CIM and IEC 61850. Hence, the relations of the SCL schema's concepts with the concepts of CIM is straightforward. On the other hand, if the harmonization is done by OWL-ontology approach, we are assuming that there are independent UML models for the independent concepts of each standard. Hence, the selection of the subsets of these standards will be done for each UML model. This means that a profile is required for each ontology. However, the relation of these profiles will be managed by the OWL-axioms of the harmonization.

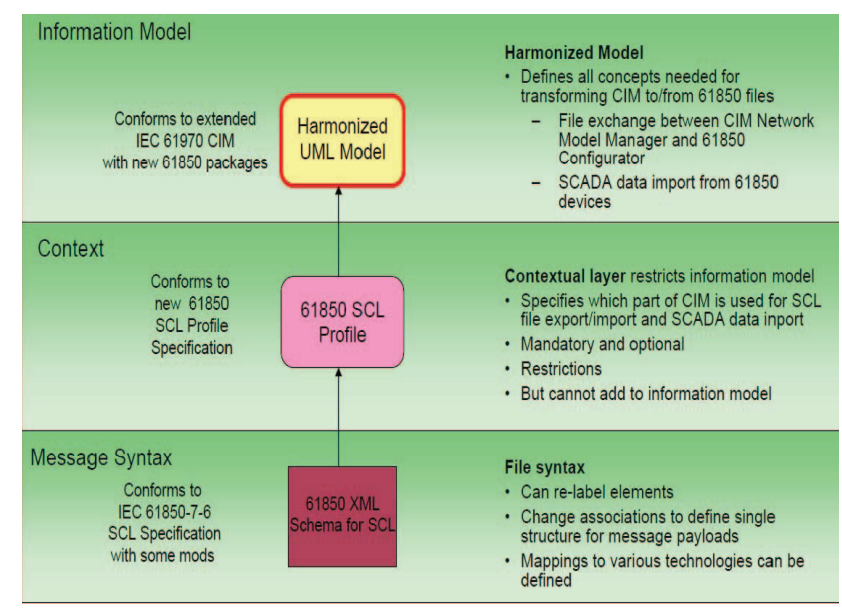

Fig. 9. A vision for SCL file Export and Import from a unique harmonized UML model.

One expected feature of the harmonized semantic data model is its capacity for generating SCL files. As we already commented, SCL files are the core for the configuration of intelligent electronic devices. In the case that there is a unique UML model, these files can be exported/imported from a profile of this UML model. This idea is depicted in Fig. 9. 
TABLE I

APPROACHES FOR HARMONIZING IEC 61850 AND CIM

\begin{tabular}{|l|l|l|}
\hline Features & $\begin{array}{l}\text { An unified UML } \\
\text { model }\end{array}$ & $\begin{array}{l}\text { An OWL-ontology } \\
\text { approach }\end{array}$ \\
\hline $\begin{array}{l}\text { Update of CIM } \\
\text { version }\end{array}$ & Rigid & Flexible \\
\hline $\begin{array}{l}\text { Managing of pro- } \\
\text { files }\end{array}$ & A unique profile & $\begin{array}{l}\text { A profile for each on- } \\
\text { tology }\end{array}$ \\
\hline $\begin{array}{l}\text { Generation of } \\
\text { SCL files }\end{array}$ & A unique mapping & $\begin{array}{l}\text { OWL - mappings be- } \\
\text { tween ontologies }\end{array}$ \\
\hline $\begin{array}{l}\text { Features for rea- } \\
\text { soning process }\end{array}$ & $\begin{array}{l}\text { Restricted to single } \\
\text { relations between } \\
\text { concepts } \\
\text { relations) }\end{array}$ & $\begin{array}{l}\text { Rich variety of re- } \\
\text { lations and axioms } \\
\text { (OWL-axioms). }\end{array}$ \\
\hline
\end{tabular}

On other hand, if the harmonization is managed by OWLontologies, one require to map a CIM profile into IEC 61850 profiles which contains the concepts of SCL schema. This means the generation of the SCL files require using the OWL axioms which specify the relations between the classes of CIM and IEC 61850.

In the conceptualization of Smart Grid, one is assuming that the Smart Grid has capacities for been reactive and proactive in the presence of abnormal events. This ability suggests that a smart grid has capabilities for performing reasoning process. By reasoning processes, we mean that the smart grid is able to infer knowledge. Hence, one can expect that the harmonized semantic model between CIM and IEC 61850 has to offer mechanisms for capturing knowledge. In this sense, the harmonization of these standards by using a unique UML model is limited to express relations between concepts. On the other hand, the approach of harmonization based on OWL ontologies offers a rich approach for capturing and inferring knowledge.

In general terms, we can see that the two approaches for harmonizing IEC 61850 and CIM have pros and cons, Table I summarizs some features of each approach.

\section{CONCLUSIONS}

In the vision of interoperability of a smart grid, one can find several industrial standards which offer different data interoperability levels. However, these standards cover different data domain with large overlaps among them. This scenario has motivated to international organizations such as NIST to support research for the harmonization of some standards.

Since CIM ( $($ III) and IEC 61950 ( $(I V)$ are two of the most accepted standards of the IEC's standards, the EPRI has supported intensive research for harmonizing these standards. The main strategy for harmonizing these standards is based on semantics data models $(\S \mathrm{V})$. These semantic data models have been defined by using UML models. By using UML models, the harmonization can be done in a high level of abstraction. Since these UML models implicitly define single ontologies, the harmonization can be regarded as a problem of harmonization of ontologies. To use only UML models or a combination of UML models and OWL ontologies for harmonizing CIM and IEC 61950 gives different pros and cons in the vision of smart grids $(\S \mathrm{V})$.
To regard semantics data models such OWL-ontologies opens the possibility for performing reasoning processes via Description Logic Formalisms. On the other hand, one can take advantage of semantics web technology such as SPARQL $^{8}$, SRWL $^{9}$, description logic reasoners ${ }^{10}$.

\section{ACKNOWLEDGMENT}

This work has been supported by the ENERGOS Project funded by the Spanish MEC (ENERGOS TIN2009-00000). The authors would like to acknowledge the contributions of their colleagues from the ENERGOS consortium (http://innovationenergy.org/energos). The views expressed in this paper are not necessarily those of the ENERGOS consortium.

\section{REFERENCES}

[1] D. V. Dollen, "Report to NIST on the Smart Grid Interoperability Standards Roadmap - Post Comment Period Version," Electric Power Research Institute (EPRI), Deliverable, 2009.

[2] IEC Smart Grid Standardization Roadmap, IEC - International Electrotechnical Commission Std. Ed. 1.0 - 2009-12, June 2010.

[3] Microsoft, "Microsoft Power and Utilities - Smart Energy Reference Architecture (SERA) ,"www.Microsoft.com/Utilities, October 2009.

[4] J. Hughes, "Harmonization of IEC 61970, 61968, and 61850 Models," Electric Power Research Institute (EPRI), Tech. Rep. 1012393, December 102006.

[5] D. Becker, "Harmonizing the International Electrotechnical Commission Common Information Model (CIM) and 61850," Electric Power Research Institute (EPRI), Tech. Rep. 1020098, May 2010.

[6] S. Rusell and P. Norvig, Artificial Intelligence: A Modern Approach. Pretice Hall Series in Artificial Intelligence, 2003.

[7] C. Baral, Knowledge Representation, Reasoning and Declarative Problem Solving. Cambridge: Cambridge University Press, 2003.

[8] F. Baader, D. Calvanese, D. McGuinness, D. Nardu, and P. PatelSchneider, Eds., The Description Logic Handbook - Theory, Implementation and Applications. Cambridge University Press, 2003.

[9] IEC, "IEC 61970 Energy management system application program interface (EMS-API) - Part 301: Common Information Model (CIM) Base," Standard of the International Electrotechnical Commission, November 2003.

[10] —, "IEC 61968 Application integration at electric utilities - System interfaces for distribution management - Part 11: Common information model (CIM) extensions for distribution ," Standard of the International Electrotechnical Commission, 2010.

[11] SISCO, "Overview and Introduction to the Manufacturing Message Specification (MMS)," System Integration Specialists Company, Inc. (SISCO), Tech. Rep., 2005.

[12] M. Adamiak, D. Baigent, and R. Mackiewicz, "IEC 61850 Communication Networks and Systems In Substations: An Overview for Users," GE Digital Energy and SISCO, Tech. Rep., 2004.

[13] IEC, "Communication networks and systems for power utility automation Part 6: Configuration description language for communication in electrical substations related to IEDs," Standard of the International Electrotechnical Commission, December 2009 - Edition 2.

[14] D. Becker, "Reference Manual for Exchanging Standard Power System Dynamic Models - Based on the IEC 61970 Common Information Model (CIM)," Electric Power Research Institute (EPRI), Tech. Rep. 1020200, September 2009.

[15] L. King, "The common information model for distribution - an introduction to the cim for integrating distribution applications and systems," Electric Power Research Institute (EPRI), Tech. Rep. 1016058, November 2008 .

\footnotetext{
${ }^{8}$ http://www.w3.org/TR/rdf-sparql-query/

${ }^{9}$ http://www.w3.org/Submission/SWRL/

${ }^{10} \mathrm{An}$ example of a description logic reasoner is PELLET: http://clarkparsia.com/pellet/
} 